\title{
On the Reduction of Grounding to Essence
}

\author{
Pablo Carnino \\ Department of Philosophy, University of Geneva
}

\begin{abstract}
In a recent article, Fabrice Correia explores the project of reducing the notion of grounding to that of essence. He then goes on to provide several candidate definitions and test each of them against a number of objections. His final take on the situation is, roughly, that two of the definitions can handle all of the considered objections. The aim of this paper is to re-evaluate Correia's conclusions in the light of two sources of insights: Firstly, I will argue that one of the objections treated by Correia has been somewhat underestimated, and that it still constitutes a threat against definitions of grounding in terms of essence. Secondly, there are at least two further objections that should be considered by the advocate of such definitions. As I will show, one of them can be neutralized; but the other one is more serious and suggests a clear dialectical edge to an operationalist definition.
\end{abstract}

Keywords: grounding, essence, necessity

\section{Introduction}

A famous notion of metaphysics tells us what a thing is. Speaking of the aspects that make a thing the thing it is, or that determine its identity, amounts to speaking about the essence of the thing in question. It is typically thought, for instance, that Aristotle is both human and bearded. But while his being bearded is not part of his nature, or essence, his being human is. Another way to put this is to say that being human makes him the very thing he is. The general idea behind this notion is that some properties of things are more intimately attached to the thing than others. Note that none of these considerations is meant as an attempt to fully define essence. Rather, they should serve to illustrate an intuitive understanding of the notion. I remain neutral here on whether the notion of essence can be adequately defined or analysed in terms of other notions and focus on how, given our superficial

Corresponding author's address: Pablo Carnino, Département de Philosophie, Université de Genève, 2, rue de Candolle, $\mathrm{CH}-1211$ Genève, Belgium. Email: pablo.carnino@unige.ch. 
understanding and intuitions on what essence is, the notion may be used to define a distinct and also important notion of metaphysics.

A perhaps less famous but recently very researched notion of metaphysics expresses the quite common philosophical idea that certain facts obtain in virtue of other facts. For instance, it is often said that moral facts obtain in virtue of non-moral (or natural) facts. When one wonders which facts obtain in virtue of which, one is wondering what grounds what. ${ }^{1}$ Much theorizing has been going on about the notion of grounding lately and the orthodoxy has it that the notion must be embraced as a primitive, for it cannot be analysed in terms of any other notion. This claim was put into question by Correia (2013) in a recent article in which he presents a number of candidate definitions of grounding in terms of essence. In the end, he does not officially endorse any of them but he still claims that two of them are good enough to resist all of the objections that he considers. I will argue that one of the objections has been underestimated by Correia and that a reshaped version of it is immune to Correia's criticism. Then I will raise two kinds of objections that Correia does not consider, the first one can seemingly be resisted by the two definitions, as they stand. But, the second is more worrisome and, as I will suggest threatens one of the definitions more seriously thus giving an edge to the other one.

Before getting to the heart of the definition, one might wonder: why at all try to define grounding in terms of essence? Let me provide some reasons that suggest that such a project might be worth pursuing. Of course, those are merely pro tanto reasons and should not be read as the premises of a deductive argument aiming to establish that one ought to analyse grounding in terms of essence.

The first thing that suggests that the notions are connected is their respective tie to metaphysical necessity. On the one hand, essence is systematically connected with necessity in the following way: if an object has a property essentially, then it also has that property as a matter of metaphysical necessity. On the other hand, whenever $x$ grounds $y$, the conditional if $x$ then $y^{\prime}$ holds as a matter of metaphysical necessity. ${ }^{2}$ That is, both notions entertain a form of systematic connection with metaphysical necessity. Besides, many metaphysicians have suggested one way or another, that grounding is connected to essence. Very roughly speaking, it seems to me that these philosophers are tempted by the idea that, provided one wants explanatory chains to bottom out somewhere, statements of definitions, or essences, are

I will use 'grounding' in an artificially narrow sense to target metaphysical grounding.

2 This claim about grounding, though often assumed, has not gone entirely unchallenged. For a discussion of it, see (Leuenberger 2014), and (Skiles forthcoming). 
ideal candidates for such end points. ${ }^{3}$ Of course, this is quite distinct from and does not entail the view that the explanatory claims in question should be understood in definitional terms, i.e. that one should define grounding in terms of essence.

Here is yet another thought that supports the strategy of using essence to understand grounding. Many cases of grounding seem to draw upon connections between the respective natures of the properties involved. For instance, there is a connection between the nature of redness and the nature of coloredness, because the former is a determinate of the latter, which is a determinable. Furthermore, that very connection seems to somehow lie behind the systematic grounding claims whereby whenever an object is red, it is colored because it is red, that is, the fact that it is colored obtains in virtue of the fact that it is red. It goes without saying that accepting these systematic connections is not tantamount to endorsing a definition of grounding in terms of essence. Kit Fine (2012, 80), for instance, accepts the links in question and rejects any attempt of defining grounding in terms of essence. However, if there is such a systematic connection, anyone rejecting the holding of a definiens-definiendum relation between the two notions should be able to otherwise account for the pattern. Similarly, holding that knowledge systematically entails belief and that neither knowledge nor belief should be defined in terms of the other seems like a delicate position on the face of it; unless of course, one can explicate why knowledge and belief are so tied. Now let us leave general motivations in the background, and turn to the definitions at hand.

\section{A Working Definition}

Before I introduce any definition, it is worth mentioning the distinction between an operationalist view of grounding and a predicationalist view of grounding. According to operationalists, grounding should ultimately be expressed in terms of a sentential operator flanked by sentential expressions, as in: 'Sam is sad because his brain is in such and such microphysiological state.' On the other hand, predicationalists use a relational predicate flanked by terms for facts or propositions to express grounding, as follows: 'The fact that Sam is sad is grounded in the fact that his brain is in such and such microphysiological state. An important difference to be noted is that only the predicationalists are committed to an ontology of facts, or the like. ${ }^{4}$ As we

${ }^{3}$ Here are some of the papers in which links between essence and grounding are explored: (Audi 2012), Dasgupta (Manuscript), (Fine 2012), Dorr (manuscript), the latter is not explicitly about essence but may easily be understood as exploring a notion in the vicinity.

${ }^{4}$ Strictly speaking, the predicationalist grammar could be adapted to exploit propositions instead of facts. But, as things stand in the relevant literature, it is paradigmatic of a com- 
will see, this may turn out to be quite important in the face of certain objections. Thus, even if the two definitions that I will be concerned with are, if you will, the predicationalist and the operationalist faces of the same coin, they are not necessarily on a par with respect to all objections. In particular, I will suggest that the operationalist definition fairs better against one of the persisting objections. In the mean time and for expository purposes, let us start with the predicationalist definition:

$\mathrm{P}_{\text {def }} f$ is grounded in $f_{i}, f_{i i}, \ldots$ iff (def)

(i) $f_{i}$ obtains \& $f_{i i}$ obtains $\& \ldots$, and

(ii) It is part of the nature of $f$ that: if $f_{i}$ obtains \& $f_{i i}$ obtains $\&$... then $f$ obtains.

More prosaically: the fact $f$ is grounded in the collection of facts $f_{i}, f_{i i}$, ... just in case the collection of facts $f_{i}, f_{i i}, \ldots$ obtains and it is part of the nature of $f$ that if the collection obtains then so does $f$. A noticeable trait of this definition is that it exploits cases of objectual essence that are somewhat different from the ones one might be used to. Indeed the object that is considered here is no typical object, it is a fact, and one might be worried that such peculiar entities do not have essential properties. My impression is that such a worry is unjustified, for our intuitions about certain properties of facts run quite similar to our intuitions about essential properties of other objects. Take Aristotle for instance: as I said earlier, he is both bearded and human but one of these properties seems to be more intimately connected with the man, or so goes the essentialist intuition. Now take the fact that Obama is the president of the United States: this fact has the property of being mentioned in this paper and the property of involving Obama but one of these properties clearly seems more intimately connected with that very fact. Indeed, it seems that if it failed to be mentioned here it would still be the same object, but if it failed to involve Obama it would no longer be the same thing. Thus, there seems to be little reason to deny that facts have essential properties once one welcomes facts as objects into one's ontology and agrees that objects have essential properties. As mentioned earlier, this does not have to be the case for operationalists. The notion analogous to the essence of a fact for the operationalist definition is what Correia (2013, forthcoming) calls "alethic essence". But I shall come back to it later: for the time being and for clarity's sake, working with the predicationalist definition will do.

It is not hard to see that some of the results of this definition will be perfectly acceptable by essentialist's standards. For instance, the very widely 
accepted thought that $f$ grounds ( $f$ or $g$ ) will entail that (i) $f$ obtains, which was already guaranteed by the factivity of grounding, and that (ii) it is part of the nature of $(f$ or $g)$ that if $f$ obtains then so does $(f$ or $g)$. If the disjunctive fact is to have a nature, as I previously argued, the cited conditional seems ideally built to figure in it. Indeed, it seems that ( $f$ or $g$ ) would hardly be the same fact if it were not such that if $f$ where to obtain then it would obtain as well; and of course, were it not the same fact, it would a fortiori not be the same object either. To take another example, suppose the fact that Sam's action maximizes utility grounds the fact that Sam's action is right. Our definition would entail that (i) the fact that Sam's action maximizes utility obtains, which as we saw is given by the hypothesis, and that (ii) it is part of the nature of the fact that Sam's action is right that if the fact that Sam's action maximizes utility obtains then the fact that Sam's action is right obtains as well. I do not think there is any problem with this, once granted the quite controversial assumption that facts about an action's being right are grounded in facts about utility maximization in the way that we assumed here. Of course, a similar example would work for any further and presumably more accurate way of specifying the grounds of rightness facts.

A kind of counter-example might come to the reader's mind here. She might say: what if there is a world in which my action maximizes utility but is not right? One might or might not want to grant such a possibility, be it as it may, the case in question functions as a counter-example not just to $\left(\mathrm{P}_{d e f}\right)$ but to any necessitarianist understanding of grounding, that is, it threatens grounding as it is most typically understood. The necessitarianist can diffuse the threat in at least two ways: first she can deny the mere possibility of a change in the normative without any change in the non-normative. After all, such a possibility is contrary to the very widely accepted thesis according to which the moral supervenes upon the non-moral. ${ }^{5}$ Secondly, she could also deny that the maximization of utility by the action grounds-in the appropriate sense-the action's being right. Here, she could rely on a distinction between metaphysical and normative grounding and say that the grounding claim in question does not belong to the class of relations that we originally set to analyse with our definition, i.e. metaphysical grounding. ${ }^{6}$

5 The thought here is not that supervenience of the normative upon the non-normative entails grounding necessitarianism. But, merely that supervenience allows us to cast away the problematic example at hand, i.e. one in which there are normative differences without any difference in the non-normative.

${ }^{6}$ Such a distinction is hinted at by Fine (2012), however this philosophical territory remains relatively unexplored. And, as I said, my discussion focuses on metaphysical grounding, I hence will not concern myself with the connection between the varieties of necessity and the varieties of grounding relations. 


\section{An Underestimated Objection}

In his seminal "Guide to Ground", Kit Fine (2012) puts forward several objections against defining grounding in terms of essence.

One of them runs basically as follows, for any object $x$ that exists at a time:

(A) It is part of the nature of $x$ that $x$ exists simpliciter iff $x$ exists at a time

The worry being that $(\mathrm{A})$ would presumably, given $\left(\mathrm{P}_{d e f}\right)$, entail a case of symmetric grounding, whereby the fact that $x$ exists at a time would ground the fact that $x$ exists simpliciter and vice versa. ${ }^{7}$ But, as Correia rightfully points out, the alleged result of symmetric grounding that Fine want to use as a reductio does not follow from his remark (A), nor does it follow from (A) combined with our $\mathrm{P}_{d e f}$. Indeed, the objection requires two further claims:

(B) It is part of the nature of the fact that $x$ exists simpliciter that if the fact that $x$ exists at a time obtains then the fact that $x$ exists simpliciter obtains.

and

(C) It is part of the nature of the fact that $x$ exists at a time that if the fact that $x$ exists simpliciter obtains then the fact that $x$ exists at a time obtains

that do not follow from (A). Clearly, (A) is an essential attribution about $x$ itself, while (B) and (C) are essential attributions about two distinct facts involving $x$. Once that has been noticed it is hard to see how (B) and (C) can follow from (A), as a matter of fact they do not. Correia presses the point by showing that the more general principle that allows inference from

$\left(\mathrm{G}_{a}\right)$ it is part of the nature of $x$ that $\mathrm{F}(x)$ iff $\mathrm{G}(x)$

to

$\left(\mathrm{G}_{b}\right)$ it is part of the nature of the fact that $\mathrm{F}(x)$ that if the fact that $\mathrm{G}(x)$ obtains then the fact that $\mathrm{F}(x)$ obtains

7 Cases of symmetrical grounding are problematic given that grounding is widely taken to be an explanatory connection and that explanations are typically asymmetrical. One typically wants to avoid simultaneously endorsing claims of the form ' $p$ because $q$ ' and ' $p$ because $q$. On a more authoritarian note, almost every single grounding theorist takes the notion to be asymmetrical, Correia and Fine included. Wilson (2014) and Rodriguez-Pereyra (forthcoming) are notable exceptions here. 
and

$\left(\mathrm{G}_{c}\right)$ it is part of the nature of the fact that $\mathrm{G}(x)$ that if the fact that $\mathrm{F}(x)$ obtains then the fact that $\mathrm{G}(x)$ obtains

has counterexamples. The one he gives is based on a version of the Aristotelian view of universals according to which it is part of the nature of humanity that it exists if and only if something exemplifies it. His suggestion is that one can maintain, consistently with this, that it is not part of the nature of the fact that something exemplifies humanity that if the fact that humanity exists obtains then the fact that something exemplifies humanity obtains as well. On the face of it, Correia is simply right, to wit, one can consistently maintain the "Aristotelian" instance of $\left(\mathrm{G}_{a}\right)$ and reject the corresponding instance of $\left(G_{c}\right)$. In sum, Fine was either tacitly relying on a false principle (that is, $\left(G_{a}\right)$ ), or he was simply drawing conclusions that do not follow from his premises.

However, as I will show here, in order for Fine's objection (or a version thereof) to work, no general principle is really required. All that would be needed, given $\left(\mathrm{P}_{d e f}\right)$, is two claims that are instances of $(\mathrm{B})$ and $(\mathrm{C})$, call them $\left(\mathrm{B}_{i}\right)$ and $\left(\mathrm{C}_{i}\right)$. My impression is that many such pairs of claims are rather plausible and Fine probably had at least some of those in mind when he formulated the objection. ${ }^{8}$ Take, for instance:

$\left(\mathrm{B}_{i}\right)$ It is part of the nature of the fact that Aristotle exists simpliciter that if the fact that Aristotle exists at a time obtains then the fact that Aristotle exists simpliciter obtains

and

$\left(C_{i}\right)$ It is part of the nature of the fact that Aristotle exists at a time that if the fact that Aristotle exists simpliciter obtains then the fact that Aristotle exists at a time obtains

even if $\left(\mathrm{B}_{i}\right)$ and $\left(\mathrm{C}_{i}\right)$ do not follow from any general principle, one could take them to be independently plausible, at least much more so than (B) and (C). Part of the difficulty with this objection lies in the blurriness of our essentialist intuitions regarding certain facts. Indeed, when asked whether it is part of the nature of the fact that Aristotle exists simpliciter that if the fact that Aristotle exists at a time obtains then the fact that Aristotle exists at a

${ }^{8}$ However, there is historical evidence that he did not, since he simply conceded when I presented him Correia's rebuttal of his objection at the Kit Fine Conference (Varano Borghi, summer 2013). But I do not wish to dwell on exegetical matters here, my point is simply that the objection can be made stronger than the one that Correia $(2013,285)$ " $[$ Is] not impressed about [...]." 
time obtains, one most naturally experiences uncertainty about the issue at hand; the case seems everything but clear here. Ironically, the essentialist's best response here might be that our intuitions regarding essences of facts are-in this case-too elusive.

It is clear, on the other hand, that provided $\left(\mathrm{P}_{\text {def }}\right),\left(\mathrm{A}_{i}\right)$ and $\left(\mathrm{B}_{i}\right)$ together would entail that the fact that Aristotle exists at a time is grounded in the fact that Aristotle exists simpliciter and vice versa, which is unacceptable for reasons I mentioned earlier. I do not claim to have provided sufficient support to establish that a version of Fine's objection actually holds. Rather, I hope to have shown that the objection, or a version thereof, is more serious than it has been thought to be. I will now turn to two kinds of new objections, the first kind relies on counter-examples drawn from the debate on grounding necessitarianism and the second consists in taking $\left(\mathrm{P}_{d e f}\right)$ to be viciously circular.

\section{A Contingentist Objection}

The class of objections that I want to consider in this section have not been raised or discussed by Fine or Correia. They belong to the debate on the connection between grounding and necessity. A not so surprising consequence of defining grounding in terms of essence is the thesis often referred to as necessitarianism about grounding:

$$
\begin{aligned}
& \left(\mathrm{N}_{\text {ground }}\right) \text { If } f \text { is grounded in } f_{i}, f_{i i}, \ldots, \text { then as a matter of metaphysical } \\
& \text { necessity: } \\
& \qquad\left(f_{i}, f_{i i}, \ldots \rightarrow f\right)^{9}
\end{aligned}
$$

Indeed, if links of grounding originate in the nature of facts, it is most obvious that these links will hold as a matter of necessity. Allow me to briefly remind the reader here that although Fine famously argued against the modal analysis of essence by arguing that necessity is not sufficient for essence, he still thinks, along with the vast majority of proponents of essence, that essence is sufficient for necessity. Thus, $\left(\mathrm{P}_{\text {def }}\right)$ entails $\left(\mathrm{N}_{\text {ground }}\right)$, and if there are cases of contingent grounding, those ipso-facto constitute counterexamples to $\left(\mathrm{P}_{d e f}\right) .{ }^{10}$ What I take to be the most convincing case for contingent grounding comes from accidental generalizations. ${ }^{11}$ Take the fact that

9 Of course, necessitarianism is a view about the notion of full-grounding. No one thinks that partial grounds necessitate. Take the fact that $p$ : it partially grounds the fact that $p \wedge q$, but this does not entail that $p \rightarrow(p \wedge q)$.

${ }^{10}$ And to its operationalist counterpart.

${ }^{11}$ These, along with other counter examples of a similar kind, were put forward and defended in (Skiles forthcoming). 
the tallest member of the Geneva department of philosophy is less than two meters tall, call this fact ' $f_{1}$ ', for short. It seems quite natural to think that:

\section{$\left(\mathrm{A}_{g e n}\right) f_{1}$ is grounded in individual facts $f_{i}, f_{i i}, \ldots$ about the respective size of each members of the Geneva department of philosophy}

or at least, so the objection goes.

Consider now a (not so) nearby world in which former basketball player Shaquille O'Neal is also a member of the Geneva philosophy department. The individual facts $f_{i}, f_{i i}, \ldots$ that grounded $f_{1}$ still obtain, but $f_{1}$ does not anymore. For, the new tallest member of the department, Shaquille O'Neal, is taller than two meters. Otherwise put, it is possible that there be someone taller than two meters in the department, i.e. that $f_{1}$ does not obtain while $f_{i}, f_{i i}, \ldots$ all obtain. $\left(\mathrm{P}_{d e f}\right)$ together with $\left(\mathrm{A}_{g e n}\right)$ entails that it is part of the nature of $f_{1}$ that: if $f_{i}, f_{i i}, \ldots$ obtain then so does $f_{1}$. But, if such a conditional is part of the nature of an object it should hold as a matter of necessity, as I just showed, it does not. ${ }^{12}$ Hence it is not. This given $\left(\mathrm{P}_{\text {def }}\right)$ suggests that $\left(\mathrm{A}_{\text {gen }}\right)$ is not grounded in the collection of facts about the respective size of the members of the actual department, contrary to our intuition. It is not too hard to see that many such objections can be created out of accidental generalizations of all sorts, which renders even more pressing the need for an answer.

A perhaps petty way to treat this objection is simply to say that, anyway, most grounding theorists are necessitarianists and that, besides, so is Fine who is the main opponent of $\left(\mathrm{P}_{d e f}\right)$. He appears to be in no position to appeal to this kind of counter-examples without losing more than he stands to win, so to speak. Still, given that $\left(\mathrm{P}_{d e f}\right)$ may have contingentist opponents and given how hard it is to know whether Fine would rather give up on necessitarianism than lose a potential counter-example to $\left(\mathrm{P}_{d e f}\right)$, I find it healthier to provide another way around the contingentist's counterexample.

The second way of replying to the contingentist is to say that the grounds cited in $\left(\mathrm{A}_{g e n}\right)$ are merely partial grounds. And that the notion that $\left(\mathrm{P}_{d e f}\right)$ defines is one of full grounds. In other words, something is missing in order for the facts $f_{i}, f_{i i}, \ldots$ about the respective sizes of the member of the department to fully ground $f_{1}$. One could be sceptic with respect to the idea that $\left(\mathrm{A}_{\text {gen }}\right)$ only constitutes a partial explanation. ${ }^{13}$ Indeed, in cases of partial

${ }^{12}$ If one, as is often done, conditionalizes the modal implications of the essential claim here, to the obtainment (or existence) of the corresponding fact, the conditional will not have to hold as a matter of necessity, but merely at each world where the fact obtains (or exists). However, this is of little importance since the counterexample will work indifferently.

${ }^{13}$ I am tacitly relying on the widely accepted view that grounding is tightly connected with explanation. In particular, I exploit a principle according to which if $x$ grounds $y$ then $x$ 
explanation, there is typically a part of the explanandum that remains unaccounted for. Suppose that I want to explain the fact that Kit Fine is a British philosopher and I cite the fact that Fine is a philosopher. It is clear enough that I have given only a partial explanation for the target fact. One way to confirm this is by noticing that a part of the explanandum, namely Fine's being British, is left unaccounted for. Coming back to $\left(\mathrm{A}_{\text {gen }}\right)$ one could answer the claim that it involves only partial grounds by saying that there is no part of the explanandum fact that is left unaccounted for in $\left(\mathrm{A}_{\text {gen }}\right)$. I think that such a response is na"ive for there is clearly a piece of the informative content of $f_{1}$ that is unaccounted for, although this may be less obviously so than in the case of partial explanation that involved Fine's being a philosopher. Suppose I ask a child: "Who is the tallest kid of your class?" and she answers: "I am 1.30 meters, Sam is 1.25 meters and Paul is 1.35 meters". I will naturally ask her if those are all the kids in her class. Only granted that information would I have a complete explanation of the fact that Paul is the tallest kid of the class. In sum, and coming back to our point, the part of the explanan$d u m$ that was unaccounted for is the fact that $f_{i}, f_{i i}, \ldots$ are all the members of the department. Once this is added to the grounds of $f_{1}$, however surprising the possibility of Shaq O'Neal being a member of the Geneva department of philosophy will not generate a counter-example to $\left(\mathrm{P}_{d e f}\right)$. For one of the facts listed as grounds for $\mathrm{f}_{1}$ will simply not obtain at that strange nearby world.

A notable problem with this response is that it commits us to a massive amount of totality facts, such as the fact that Sam and Paul are all kids in the class, the fact that $a, b$, and $c$ are all the chairs in the room, the fact that $a_{i}, a_{i i}, \ldots$ are all the atoms that compose this chair, etc. Worse yet, a large number of those facts will be fundamental, in the sense that they will not be grounded in further facts. These considerations are particularly serious if one subscribes to something like Sider's (2011) "purity principle" that says, roughly, that only fundamental entities should be mentioned in fundamental facts. ${ }^{14}$ For it would make fundamental all the entities that inhabit these ungrounded totality facts. So, many atoms, but also many (if not all) chairs, and even philosophy departments would turn out to be fundamental furniture of the world. It appears that the solution of totality facts may jeopardize the project of neo-Aristotelian ontology, according to which what matters in ontology is not what things there are but rather what fundamental things

explains $y$, in a specific sense of the word 'explain'. There might be room for a debate on the view; whether it holds and how it should be cashed out exactly. But it is not my goal to settle this question here.

${ }^{14}$ Fundamental facts are simply the facts that are not grounded in anything. 
there are. ${ }^{15}$ Part of the issue here can be avoided simply by rejecting things like the purity principle. One reason to give up on such a principle is that identity facts, e.g. the fact that Sam is Sam might be ungrounded. Following the purity principle and assuming that identity is reflexive, i.e. that everything is identical with itself; one would take everything to be fundamental. After all, each thing is involved in a self-identity fact, failing to provide suitable grounds for facts of the form $x=x$ would make every one of those facts fundamental and along with them the entities that they involve, i.e. all entities. ${ }^{16}$ Besides, the idea that one will find only fundamental things in fundamental facts seems to be derivative on the mistaken idea that if a fact grounds another fact then the entities involved in the former are more fundamental than those involved in the latter. There seem to be many cases where a fact grounds another fact but the entities involved are exactly as fundamental. It is tempting to say, for instance, that

1. The fact Sam is sad or Sam is happy is grounded in the fact that Sam is sad,

and that

2. The fact that I am at least two meters away from you is grounded in the fact that I am tree meters away from you.

But, since the very same things are involved in the grounds and in the grounded fact, one would have to conclude that Sam is more fundamental than itself, which is obviously nonsensical. Leaving aside links like the purity principle, one (perhaps serious) worry remains with the proposed solution. Namely, that it commits us to an extremely rich ontology of facts, and to some quite peculiar, one might even say baroque, facts.

This is one important respect in which a predicationalist definition and an operationalist definition of grounding are not on a par. Because the predicationalist has to assume an ontology of facts while the operationalist does not. Hence, the issue of an inflated ontology of facts and of baroque facts does not present itself or at least not in the same way for the operationalist. Take the following operationalist definition of grounding in terms of essence which is the straightforward operationalist counterpart of $\left(\mathrm{P}_{d e f}\right)$,

${ }^{15}$ For more on such a project, see (Schaffer 2009).

${ }^{16}$ Of course, the view that identity-facts are ungrounded is a substantial metaphysical thesis. And, as Skiles (personal communication) pointed out: one might say that if $x$ is a nonfundamental thing whose existence is grounded in facts about the $y$ s, then the fact that $x$ $=x$ is grounded in the fact that (i) everything that exists is self-identical and (ii) $x$ exists. One could claim that (i) is fundamental fact, albeit one that does not involve $x$; and if $x$ is non-fundamental, then (ii) is grounded in the relevant facts about the $y$ s. 
$\mathrm{O}_{\text {def }} p$ because $p_{i}, p_{i i}, \ldots$ iff (def.)

(i) $p_{i} \& p_{i i} \& \ldots$, and

(ii) The following conditional is part of what it is for it to be the case that $p$ : if $p_{i} \& p_{i} i \& \ldots$, then $p .{ }^{17}$

The definition exploits a new notion of essence that Correia (2012) calls "alethic" which tells us what it is for it to be the case that blah-e.g. what it is for it to be the case that Aristotle is human or it is sunny. This notion has some intuitive appeal, indeed, it is quite natural to think that it is part of what it is for it to be the case that Aristotle is human or it is sunny that: if Aristotle is human then Aristotle is human or it is sunny. The notion at hand might even run somewhat parallel to the notion of essence for a fact. After all, when one wonders what it is for it to be the case that Obama is the president of the United States of America, one might be after something similar to the essence of the fact Obama is the president of the United States of America. A notable difference is that the fact has the property of (perhaps even essentially) being a fact, while for it to be the case that Obama is the president of the United States of America does not seem to require that there be any facts. In his discussion of the connection between essence and grounding, Fine (2012) suggests that the notion of alethic essence can be made sense of, without further characterizing it. Some comments on the notion are also provided by Correia (forthcoming) in recent work, but the notion remains mainly unexploited. Providing a detailed account and defense of the notion in question falls outside the scope of this article. However, I hope that the few things mentioned help give a rough idea of how such an account can be developed. Be that as it may, I find it interesting and fruitful to wonder what metaphysical consequences the availability of such an account would have.

Given that a definition like $\left(\mathrm{O}_{g e n}\right)$ does not commit us to an ontology of facts, let us come back to the case of accidental generalizations and to the use of totality facts to ground them. In the spirit of $\left(\mathrm{O}_{\text {gen }}\right)$, one can perfectly take the operator 'because' to be flanked by sentential expressions that express propositions. This alternative grammar for grounding is free of any commitment to facts and thus free of commitment to totality facts as

${ }^{17}$ I should mention here that, as it stands, $\left(\mathrm{O}_{g e n}\right)$ is not equipped to distinguish plural grounds (e.g. $p_{i}, p_{i i}$ ) from conjunctive grounds (e.g. $p_{i} \& p_{i i}$ ). Such a failure to discriminate would be problematic since one typically wants a plurality of propositions to ground the corresponding conjunctive proposition, but not the conjunction to ground itself, for grounding is irreflexive. However, as Correia (2013) points out, this can be fixed by slightly tweaking the conditional that is used in clause (ii) of the definition. His response is at least technically appropriate in that it delivers the right results. 
well. Those can be replaced by totality propositions or even sentences, in the hope that they will be less gruesome from a metaphysical standpoint. Here is, in a nutshell, why such a change leaves us in better shape, so to speak. The present use of the words 'fact' and 'proposition' might be confusing to philosophers who take facts to simply be true propositions. At any rate, the distinction that matters here is the one between a merely conceptual entity (like, for instance, Fregean thoughts) and a worldly entity (like, for instance, Armstrongean states of affairs). Facts are literally speaking portions of the world; propositions, on the other hand, are purely representational entities: they present us the world as being such and such. Now having to accept totality-facts prompts the question: what part of the world corresponds to the fact that Sam and Paul are all the kids in the class? Is it the absence of any other kid in that particular class; and if so, where is that absence exactly? These difficult questions and others seem to arise only for the ontological category of fact. Indeed, there is nothing metaphysically mysterious about a totality proposition in itself. ${ }^{18}$

What about the fact that many of those totality propositions will be ungrounded? Is that problematic? Here are two reasons why one might think that having many totality propositions without grounds is an issue: firstly, one might want to hold on to some kind of purity principle for propositions according to which there are only fundamental entities in ungrounded true propositions. I think that the considerations that I raised for rejecting the factual version of the principle apply mutatis mutandis; secondly, one might want to defend a special version of the principle of sufficient reason (PSR) according to which no proposition should be ungrounded. But in order to be any better off, such a philosopher would have to show that she can avoid having the true totality propositions at all, and this seems to be a rather tricky task. Will she deny that Kevin Mulligan, Ghislain Guigon, ... are all the members of the Geneva philosophy department? That $a_{1}, a_{2}, \ldots$ are all the chairs in the classroom? I can hardly see how one would go about doing that. My preliminary conclusion here is that the contingentist objections, at least the ones relying on accidental generalizations, can be avoided by $\left(\mathrm{O}_{\text {gen }}\right)$ but not by $\left(\mathrm{P}_{d e f}\right)$. Given that other considerations apply quite similarly to both definitions there is a general advantage to $\left(\mathrm{O}_{\text {gen }}\right)$, provided that the appropriate essentialist notion can be worked out.

${ }^{18}$ Of course, the truthmaker maximalist will encounter analogous issues accounting for the truth of that proposition. 


\title{
5. Is the Definition Circular?
}

For the sake of grammatical simplicity I will present this objection as though it concerned the predicationist definition. However, I am convinced that both $\left(\mathrm{O}_{g e n}\right)$ and $\left(\mathrm{P}_{d e f}\right)$ can escape the objection and that the corresponding responses would be quite similar, enough so that I can afford to only present one and let the reader extrapolate from there. The objection is the following: the notion of being part of the essence of $f$ that appears in clause two of $\left(\mathrm{P}_{d e f}\right)$ 's definiens is simply grounding under another guise. ${ }^{19}$ In other words, what should be defined is in fact already present in what supposedly serves to define it. Indeed, the Finean locution for essential attribution "...true in virtue of the nature of..." is dangerously close to a very common way of expressing grounding relations, that is, the '.. in virtue of...' connective (Fine 1994). However, Fine explicitly dismisses understanding the former in terms of the latter:

\begin{abstract}
Although the form of words 'it is true in virtue of the identity of $x$ ' might appear to suggest an analysis of the operator into the notions of the identity of an object and of a proposition being true in virtue of the identity of an object, I do not wish to suggest such an analysis. The notation should be taken to indicate an unanalyzed relation between an object and a proposition. (Fine 1995a, 284)
\end{abstract}

Leaving Fine's exegesis on a side, even if, quite literally speaking, the sequence of words 'true in virtue of the nature of' does contain the sequence 'in virtue of', this-on its own-does not show that the notion referred to by 'in virtue of' when it is used to express grounding is at play in the notion referred to by 'being part of the essence of $f$ ' as it is used in $\left(\mathrm{P}_{d e f}\right)$ or by essentialists in general. There is at least one plausible way of understanding the notion of being part of the essence of $f$ which does not induce this circularity. One might think that the essence of $f$ is something like a set or collection of propositions about $f$. There would be the set of true propositions about $f$, perhaps also the set of necessary propositions about $f$, and finally the set of essential propositions about $f$. According to this way of understanding essence, what is part of it could either be a member of the set or a proposition that is somehow derived from some propositions that are members of the set. ${ }^{20}$ On such a conception of essence, there is apparently no circularity. Besides, this is not far from Fine's understanding of essence,

\footnotetext{
${ }^{19}$ My thanks go to Kevin Mulligan for raising this objection.

${ }^{20}$ The derivation in question yields the "consequential"- as opposed to constitutive-essence of the object. Fine (1995b) elaborates on this distinction. The details of the derivation at play are subject to controversies, but they should not matter too much here. More can be found on this in (Correia 2012).
} 
except that he puts emphasis on essence being a primitive connection between an object and some propositions whereby the later are true in virtue of the nature of the former. But no matter what the primitive ideology looks like in words, it connects one or more objects to one or more propositions. With this in mind, one would probably understand being part of the essence of $f$ as being amongst the propositions $q_{i}, q_{i i}, \ldots$ that are true in virtue of the nature of $f$. Now the question remains: does ' $p$ is true in virtue of the nature of ...' express a grounding relation. There are mainly two reasons to think that the Finean locution does not express grounding. One is that the locution connects an object like Aristotle and a proposition, while grounding connects facts with facts or propositions with propositions. And the second is that grounding is an explanatory connection, while the essentialist locution connects an object with one or more propositions, and an object cannot possibly explain anything. At best, the object's existing or some other proposition about the object may play the role of grounds but not the object on its own. ${ }^{21}$ Hence, our understanding of essence does not lead to the conclusion that $\left(\mathrm{P}_{d e f}\right)$ is circular.

\section{Concluding Words}

I hope to have cast some new light on the prospects of defining grounding in terms of essence, at least as far as the two definitions that I presented are concerned. I suggested that an objection that Correia had quickly dismissed is much more serious than he might have thought. In addition, I tested the results of two definitions against some new objections. It turns out that, if they can equally handle the threat of circularity, the operationalist definition is better suited to withstand the contingentist's objection. This provides a dialectical edge to the operationalist definition and, indirectly, an argument in favour of operationalism about grounding.

\section{Acknowledgments}

I wish to thank all the participants of the eidos seminar (Geneva, 14th April 2014), as well as those of the Fine conference (Varano Borghi, summer 2013) for helpful discussions on earlier versions of this paper. Many thanks also to Justin Zylstra and Tuomas Tahko for their extensive comments. Finally, I am grateful to Fabrice Correia and Alex Skiles for their invaluable feedback and support. This research was supported by the Swiss National Science Foundation and part of the Sinergia project "Grounding: Metaphysics, Science, and Logic" (Project 147685).

${ }^{21}$ This is considered as a rule by most grounding theorist, Schaffer-at least in his earliest theorizing about grounding-comes to mind as an exception. 


\section{Bibliography}

Audi, P. (2012). Grounding: Toward a theory of the in-virtue-of relation, Journal of Philosophy 109: 685-711.

Correia, F. (2012). On the reduction of necessity to essence, Philosophy and Phenomenological Research 84: 639-653.

Correia, F. (2013). Metaphysical grounds and essence, in M.Hoeltje, B.Schnieder and A.Steinberg (eds), Varieties of Dependence. Ontological Dependence, Grounding, Supervenience, Response-Dependence, Basic Philosophical Concepts Series, Philosophia, München, pp. 271-296.

Correia, F. (forthcoming). More on the reduction of necessity to essence, in M. Dumitru (ed.), Metaphysics, Meaning, and Modality: Themes from Kit Fine, Oxford University Press, Oxford.

Correia, F. and Schnieder, B. (2012). Grounding : An opinionated introduction, in F. Correia and B. Schnieder (eds), Metaphysical Grounding: Understanding the Structure of Reality, Cambridge University Press, Cambridge, pp. 1-36.

Dasgupta, S. (n.d.). The status of ground. Unpublishied manuscript.

Dorr, C. (n.d.). To be an $f$ is to be a $g$. Unpublished manucript.

Fine, K. (1994). Essence and modality, Philosophical Perspectives 8: 1-16.

Fine, K. (1995a). Ontological dependence, Proceedings of the Aristotelian Society 95: 268-290.

Fine, K. (1995b). Senses of essence, in W. Sinnott-Armstrong (ed.), Modality, Morality and Belief, Cambridge University Press, Cambridge, pp. 53-73.

Fine, K. (2012). Guide to ground, in F. Correia and B. Schnieder (eds), Metaphysical Grounding: Understanding the Structure of Reality, Cambridge University Press, Cambridge, pp. 37-8o.

Leuenberger, S. (2014). Grounding and necessity, Inquiry 57: 151-174.

Rodriguez-Pereyra, G. (forthcoming). Grounding is not a strict order, Journal of the American Philosophical Association.

Schaffer, J. (2009). On what grounds what, in D. Manley, D. Chalmers and R. Wasserman (eds), Metametaphysics: New Essays on the Foundations of Ontology, Oxford University Press, New York, pp. 347-383.

Sider, T. (2011). Writing the Book of the World, Oxford University Press, New York.

Skiles, A. (forthcoming). Against grounding necessitarianism, Erkenntnis.

Wilson, M. J. (2014). No work for a theory of grounding, Inquiry 57: 535-579. 